\title{
Supramolecular polymer formation by metal-ligand complexation: MC simulations and analytical modeling
}

\author{
Chun-Chung Chen and Elena Dormidontova \\ Supporting Information
}

\section{Solutions to the equations of chemical equilibrium}

The equations of chemical equilibrium (eqs 2 and 3 ) can be re-written in the form:

$$
\begin{gathered}
m_{1}^{2}+2 m_{1} m_{2}-2 s m_{1}+b m_{2}=0 \\
\left(1-m_{1}-m_{2}\right) m_{2}=m_{1}^{2} c
\end{gathered}
$$

where $s \equiv \mathcal{N}_{o} / \mathcal{N}_{m}$ is the fraction of oligomers to metals, $b \equiv \frac{V}{v s} \tilde{K}_{2}=\frac{V}{v s} e^{-\Delta E_{2} /(k T)+\Delta S_{2}}$ and

$$
c \equiv \frac{\tilde{K}_{2}}{\tilde{K}_{1}}=\exp \left[\frac{\Delta E_{2}-\Delta E_{1}}{k T}+\Delta S_{1}-\Delta S_{2}\right]
$$

From eq S.1 the fraction of 2:1 complexes, $m_{2}$, can be expressed via the fraction of 1:1 complexes, $m_{1}$, as

$$
m_{2}=\frac{\left(2 s-m_{1}\right) m_{1}}{\left(2 m_{1}+b\right)} .
$$

Substitution of $m_{2}$ into eq S.2 leads to the cubic equation:

$$
(4 c-1) m_{1}^{3}+[2+b(4 c-1)] m_{1}^{2}+\left[b^{2} c+4 s(s-1)+b(1+2 s)\right] m_{1}-2 b s=0
$$

which can be presented as

$$
A m_{1}^{3}+B m_{1}^{2}+C m_{1}-D=0
$$

with coefficients $A \equiv 4 c-1, B \equiv[2+b(4 c-1)], C \equiv b^{2} c+4 s(s-1)+b(1+2 s)$, and $D \equiv 2 b c$.

For $A>0$, there is only one real and positive root of $m_{1}$ for eq S.4,

$$
m_{1}=\frac{J^{1 / 3}-Q J^{-1 / 3}-B}{3 A}
$$

where

$$
\begin{gathered}
J \equiv P+\sqrt{P^{2}+Q^{3}}, \\
Q \equiv 3 A C-B^{2},
\end{gathered}
$$

and

$$
P \equiv \frac{1}{2}\left(27 A^{2} D+A B C-2 B^{3}\right)
$$

Note that when $P^{2}+Q^{3}<0, Q$ is negative and $J$ becomes a complex number $J=|J|(\cos \theta+i \sin \theta)$ with $\tan \theta=\operatorname{Im} J / \operatorname{Re} J \neq 0$ where $\operatorname{Im} J$ and $\operatorname{Re} J$ are respectively the imaginary and the real part of $J$. But, all the imaginary parts in eq S.5 cancel and $m_{1}$ remains real,

$$
m_{1}=\frac{2 \sqrt{-Q} \cos (\theta / 3)-B}{3 A} .
$$


For $A<0$, all three roots are real, but only one is between 0 and 1 ,

$$
m_{1}=\frac{2 \sqrt{-Q} \cos [(\theta+2 \pi) / 3]-B}{3 A}
$$

where $Q<0$.

In the special case $A=0 \rightarrow c=1 / 4$, the equation becomes

$$
2 m_{1}^{2}+C_{o} m_{1}-D=0
$$

where $C_{o}=\frac{1}{4} b^{2}+4 s(s-1)+b(1+2 s)$. The solution of the equation is

$$
m_{1}=\frac{\sqrt{C_{o}^{2}+8 D}-C_{o}}{2}
$$

Equations S.10-S.12 along with eq S.3 give the complete set of solutions to eqs 3 and 4 . The total degree of association is based on 2:1 ligand-metal complex formation and can be calculated as

$$
p=\frac{m_{2}}{s}
$$

The weight average molecular weight (in units of oligomer molecular weight) can be estimated as (see Ref. 25)

$$
M_{W}=\frac{1+p}{1-p}
$$

We note that this expression is only an approximation since it does not account for ring formation or rigidity of complexation (which may change the average molecular weight). 